\title{
Solar Modulation of Galactic Cosmic-Ray Electrons Measured with CALET
}

\author{
Shoko Miyake*1, Yotaro Migita ${ }^{2}$, Yoichi Asaoka ${ }^{2}$, Yosui Akaike ${ }^{3,4}$, Shoji Torii ${ }^{2}$, Toshio \\ Terasawa $^{2}$, Ryuho Kataoka ${ }^{5}$, and Kenichi Sakai ${ }^{3,4}$ \\ ${ }^{1}$ National Institute of Technology (KOSEN), Ibaraki College, Ibaraki, Japan \\ ${ }^{2}$ Waseda University, Tokyo, Japan \\ ${ }^{3}$ University of Maryland, Baltimore, MD, USA \\ ${ }^{4}$ NASA Goddard Space Flight Center, Greenbelt, MD, USA \\ ${ }^{5}$ National Institute of Polar Research, Tokyo, Japan \\ E-mail: miyakeskdee.ibaraki-ct.ac.jp
}

\section{for the CALET Collaboration ${ }^{\dagger}$}

\begin{abstract}
The CALorimetric Electron Telescope (CALET) installed on the International Space Station has multiple event trigger modes for measuring high-energy particles and gamma rays. The observations of the low-energy cosmic-ray (CR) electrons (electrons + positrons) in an energy region from $1 \mathrm{GeV}$ to $10 \mathrm{GeV}$ have been successfully performed by a low-energy shower trigger mode working in the geomagnetic polar regions. The continuous measurements of the low-energy CR electrons may provide a crucial key to the understanding of the solar modulation of the galactic cosmic rays. Here we have analyzed the low-energy CR electrons measured by CALET over the past three years to investigate the solar modulation of the CR electrons. We have obtained the continuous variation of the low-energy electron flux increasing as time passes, which have been expected from a recent weakening solar cycle. We have also confirmed that there are additional small fluctuations in the flux, that has a potential to be explained by the effects of the interplanetary coronal mass ejections or the co-rotating interaction region of the solar wind.
\end{abstract}

36th International Cosmic Ray Conference -ICRC2019-

July 24th - August 1st, 2019

Madison, WI, U.S.A.

\footnotetext{
* Speaker.

${ }^{\dagger}$ for collaboration list see PoS(ICRC2019) 1177
} 


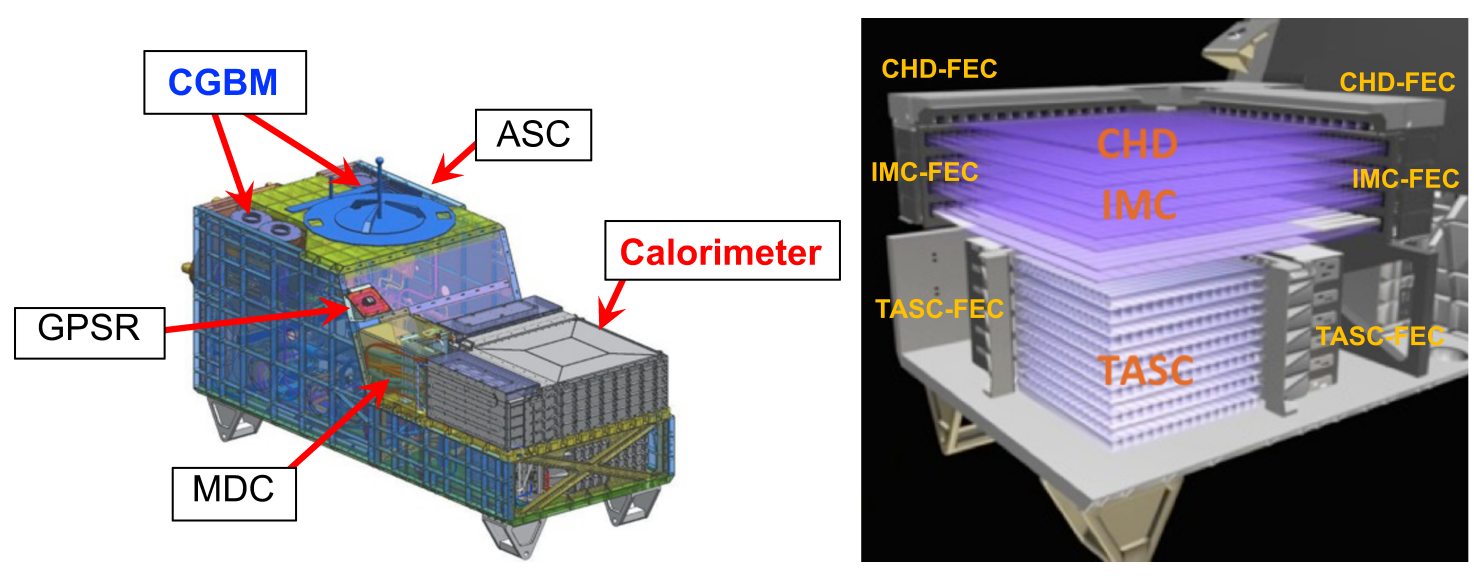

Figure 1: (Left panel) CALET instrument package showing the calorimeter, the CALET gamma-ray burst monitor (CGBM), the mission data controller (MDC), the GPS receiver (GPSR), and the advanced stellar compass (ASC). (Right panel) CALET calorimeter, which consists of a charge detector (CHD), an imaging calorimeter (IMC), and a total absorption calorimeter (TASC). The resulting output of each detector is sent to the front-end circuit (FEC).

\section{Introduction}

The CALorimetric Electron Telescope (CALET) is a Japanese-Italian-US international mission for precise measurements of cosmic-ray (CR) electrons (electrons + positrons) from $1 \mathrm{GeV}$ to $20 \mathrm{TeV}$, gamma rays from a few $\mathrm{GeV}$ to $10 \mathrm{TeV}$, and nuclei with $Z=1$ to 40 from a few $10 \mathrm{GeV}$ up to $\sim 1 \mathrm{PeV}$. The instrument was installed on the Japanese Experiment Module-Exposed Facility of the International Space Station (ISS) in August 2015, and since the beginning of operation in October 2015, continuous observations have been accomplished without any serious problem at present. Many of science goals of CALET including the investigation of possible nearby CR sources and dark matter signatures by the high-precision measurement of electron spectrum have been performed by a high-energy (HE) shower trigger mode for measuring high-energy electrons with energies above $10 \mathrm{GeV}$ and the other high-energy shower events [U, 口, []]. In addition to the HE shower trigger mode, CALET has a low-energy (LE) shower trigger mode working at high geomagnetic latitudes that can measure the low-energy $\mathrm{CR}$ electrons in the energy region from 1 $\mathrm{GeV}$ to $10 \mathrm{GeV}$ [困, []. In this study, we have analyzed flight data obtained by the LE shower trigger mode from October 12, 2015 to April 30, 2019.

\section{CALET Instrument}

CALET has a field-of-view of $\sim 45^{\circ}$ from the zenith and an effective geometrical factor of $\sim$ $1040 \mathrm{~cm}^{2} \mathrm{sr}$ for high-energy electrons. The instrument of CALET consists of the main calorimeter and the CALET gamma-ray burst monitor (CGBM) subsystems as shown in a left panel of Figure 1. Details of the main calorimeter is shown in the right panel of Figure 1, which consists of a charge detector (CHD) for identifying the charge of an incident particle, an imaging calorimeter (IMC) for reconstructing the track of an incident particle and for imaging an early shower profile with a fine resolution, and a total absorption colorimeter (TASC) for absorbing the entire energies 


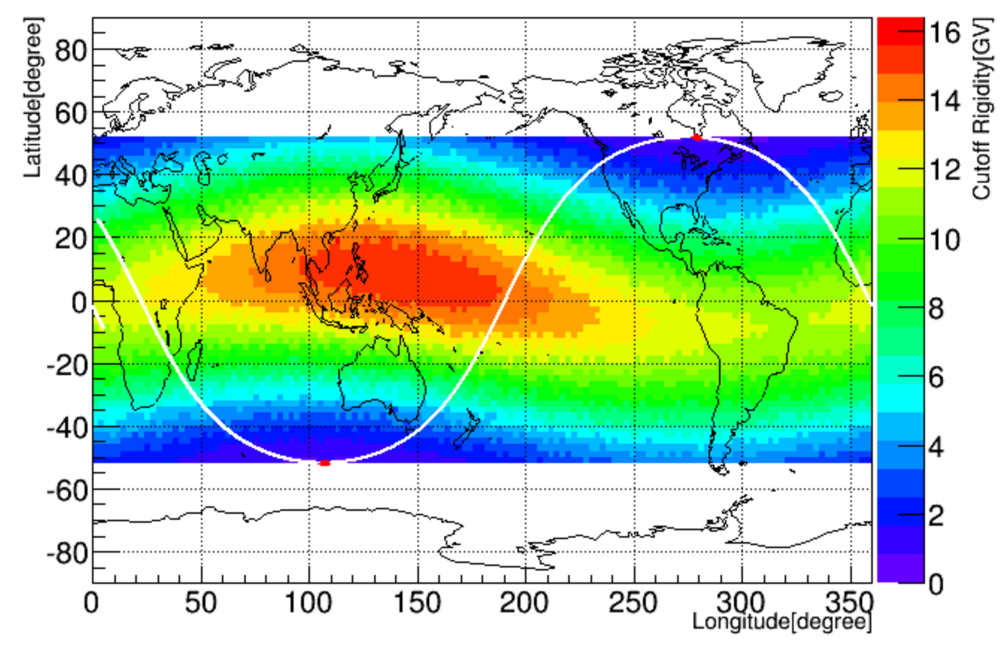

Figure 2: Geomagnetic vertical cutoff rigidity map in the area of ISS orbit (white curve).

of electromagnetic shower particles and for identifying electrons from hadrons using differences of those shower developments. The CHD is composed of two crossed layers of 14 plastic scintillator paddles with dimensions of $450 \mathrm{~mm}$ long $\times 32 \mathrm{~mm}$ wide $\times 10 \mathrm{~mm}$ thick. The IMC is composed of eight $x$-y layers of $448 \mathrm{~mm}$ long $\times 1 \mathrm{~mm}$ square cross section scintillating fibers (SciFi) interleaved with tungsten plates. The first five tungsten plates have 0.2 radiation length $\left(X_{0}\right)$ in thickness and the last two plates have $1.0 X_{0}$. The total thickness of the IMC is equivalent to $3 X_{0}$. The TASC consists of twelve crossed layers of 16 lead tungstate (PWO) logs with $326 \mathrm{~mm}$ long $\times 19 \mathrm{~mm}$ wide $\times 20 \mathrm{~mm}$ tall, and its total thickness corresponds to $27 X_{0}$. The thick calorimeter with $30 X_{0}$, which is equivalent to $\sim 1.3$ proton interaction lengths, allows it to completely absorbs the electron shower energy even in the TeV energy range.

\section{Data Analysis Strategy}

We have analyzed flight data obtained by the LE shower trigger mode for 1297 days from October 12, 2015 to April 30, 2019. Figure $\square$ shows a geomagnetic vertical cutoff rigidity map for the ISS orbit. In order to obtain the low-energy particle events efficiently, the LE shower trigger mode is active only at high geomagnetic-latitudes where the maximum cutoff rigidity is $5.0 \mathrm{GV}$ or following a low-energy gamma ray burst trigger by CGBM. It means that the LE shower trigger mode works $\sim 2$ times for $\sim 90 \mathrm{sec}$ in one orbit of ISS. We obtain a total observational live time of 28,213 hours and about 55 million events of low-energy electron candidates. The procedure to analyze the low-energy electrons obtained by LE shower trigger mode is as follows.

\subsection{Monte Carlo Data}

A Monte Carlo (MC) data was used to simulate physics processes and detector signals for the event selection and the energy reconstruction. The $\mathrm{MC}$ event samples consist of downgoing electrons and protons produced isotropically on the surface of a sphere with a radius of $78 \mathrm{~cm}$ 


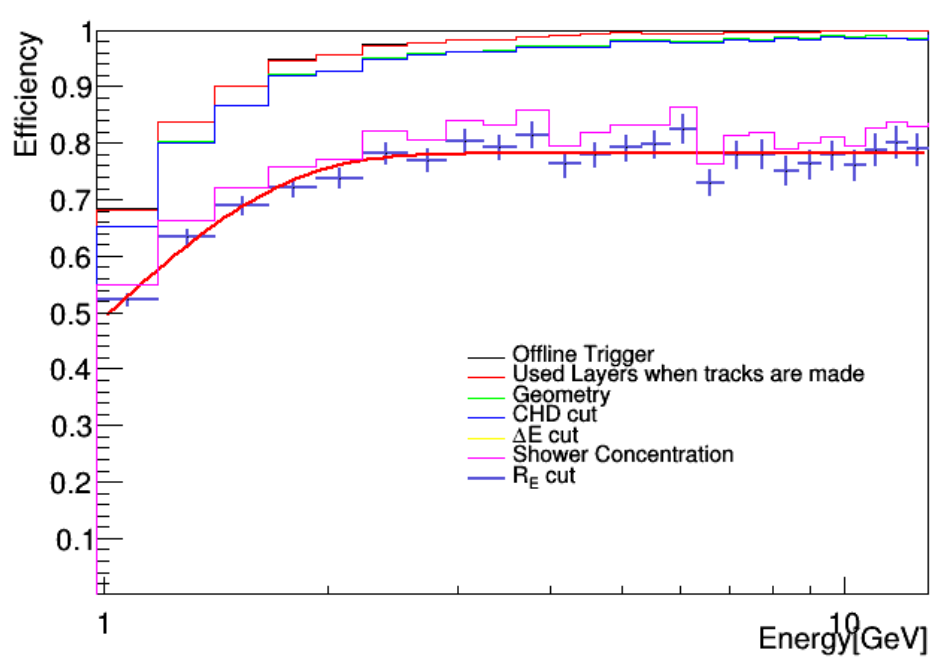

Figure 3: Efficiency of the low-energy electrons.

which totally encloses the instrument. The interactions of secondary particle generated in the

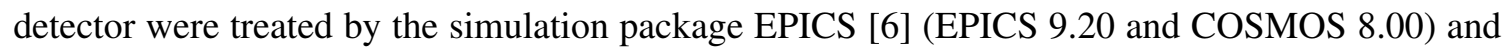
the hadron interaction was calculated by using the DPMJET-III model.

\subsection{Event Selections}

In order to minimize and accurately subtract proton contamination in the sample of electron candidates, we applied the following event selections: (1) an off-line trigger confirmation using energy threshold of lower two layers of IMC and TASC top layer, (2) a track quality cut based on the tracking method using the Kalman filter to ensure reconstruction accuracy, (3) a geometrical condition in which the reconstructed track traverse the instrument from the CHD top layer to the TASC bottom layer, (4) a charge selection using the CHD, (5) an energy deposit at the IMC bottom layer, (6) an energy concentration at the IMC bottom layer, and (7) a lateral shower development at the TASC top layer. These procedures are almost identical to that for the event selections of highenergy electrons obtained by the HE trigger mode [U], except that we just use TASC top layer and IMC bottom layer to prevent proton contamination since the low-energy electrons cannot penetrate all layers of the TASC. The combined efficiency of these selections for the low-energy electrons is over $70 \%$ in the energy region above $2 \mathrm{GeV}$, as shown in Figure [3].

\subsection{Energy Reconstruction}

In order to reconstruct the true energy of the low-energy electrons, we evaluate an energy correction function using the electron MC data after the event selections. The observed energy of the low-energy electron is defined as the sum of the energy deposit in the IMC and top three layers of the TASC to prevent adding the noise at lower layers of the TASC where the low-energy electrons cannot penetrate. The correction function is evaluated as the average ratio of the true energy to the observed energy. 


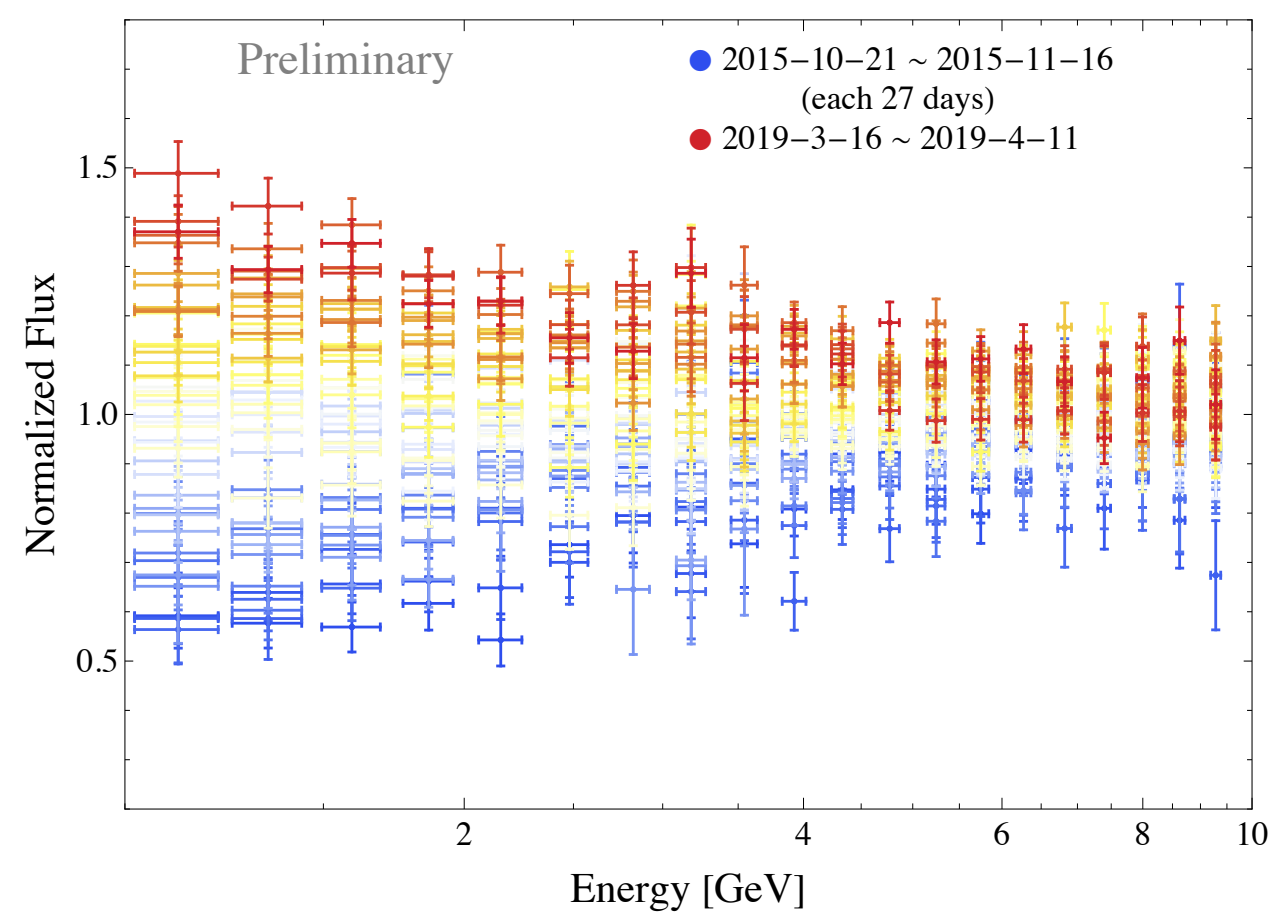

Figure 4: Normalized differential energy spectra of the CR electrons in the energy region from $1 \mathrm{GeV}$ to $10 \mathrm{GeV}$ observed by CALET in each 27-days superimposed in color sequences (blue $\sim$ red) in the period from October 2015 to April 2019. The fluxes in each energy are normalized with the mean value of the flux during the whole periods.

\section{Flux of the Low-Energy Electrons}

The differential flux $\Phi(E)$ between $E$ and $E+\Delta E(\mathrm{GeV})$ with bin width $\Delta E(\mathrm{GeV})$ is given by the following formula,

$$
\Phi(E)=\frac{N(E)}{\Delta E \cdot \varepsilon(E) \cdot S \Omega \cdot T_{L}},
$$

where $\Phi(E)$ is expressed in $\mathrm{m}^{-2} \mathrm{sr}^{-1} \mathrm{sec}^{-1} \mathrm{GeV}^{-1}, N(E)$ is the number of low-energy candidates in each energy bin, $\varepsilon(E)$ is the electron efficiency in each energy bin, $S \Omega\left(\mathrm{m}^{2} \mathrm{sr}\right)$ is the geometrical acceptance, and $T_{L}$ (sec) is the observational live time.

To minimize the impact of the geomagnetic cutoff of the low-energy electrons, we calculate the low-energy electron spectrum by combining the fluxes evaluated from the electron candidates measured in several regions with different threshold of the geomagnetic cutoff rigidity. In this study, we calculated the geomagnetic cutoff rigidity with Stoermer cutoff rigidity function (e.g. Cooke et al., 1991 [四]). Although this function may be an oversimplified model, it is assumed that this introduces no serious problems in our results since we combined the fluxes of each energy by considering a sufficiently tight threshold of the geomagnetic cutoff rigidity. In the detailed analysis in the near future, we will adopt the effective cutoff rigidity considering an empirical geomagnetic field, such as IGRF2012 and TS05 models, by calculating the gyromotion of the CRs using the standard technique for the time-reversed Lorentz trajectory of the negatively charged antiprotons [四]. 


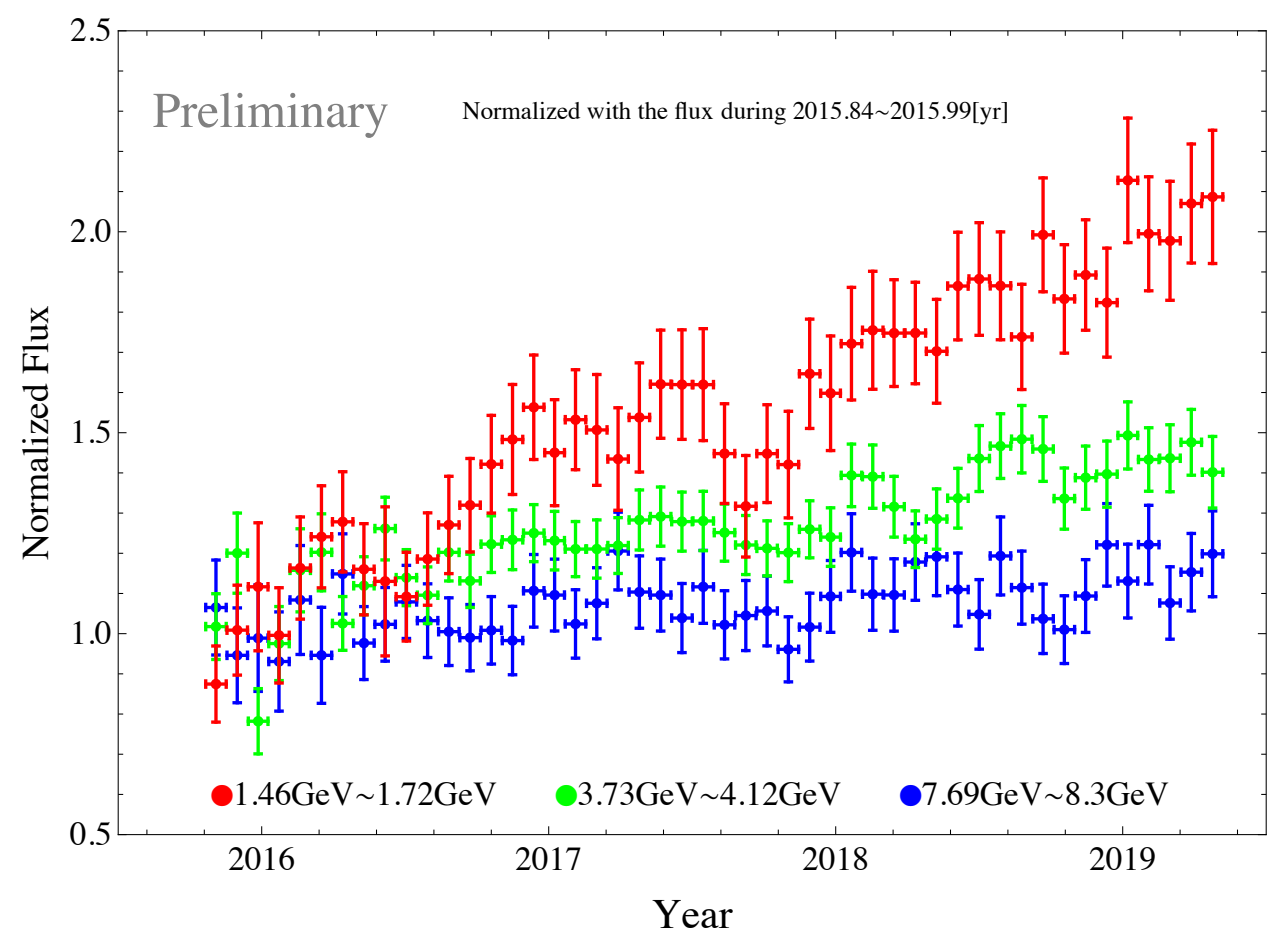

Figure 5: Time profile of the normalized flux of the low-energy electrons from October 2015 to April 2019. The fluxes in each energy are normalized with the mean value of the first 3 fluxes.

Figure 1 shows the normalized energy spectra of the low-energy electrons measured with LE trigger of CALET. We can find that the flux of the low-energy electrons have gradually increased with time from October 2015 to April 2019. It is explained by a recent solar cycle heading towards the solar minimum. The feature in which larger modulation of a few $\mathrm{GeV}$ electrons than that the electrons with higher energy also be consistent with the basic feature of the solar modulation

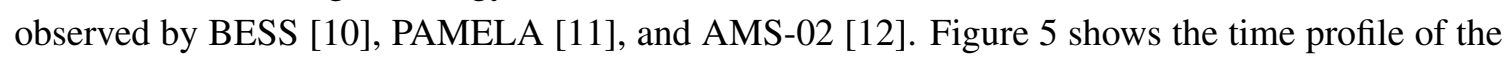
normalized flux of the low-energy electrons observed by CALET. In addition to the yearly based solar modulation, we can find the small disturbances of the flux, for example during the second half of 2017. These short-period disturbances may have some relationship with the interplanetary coronal mass ejection and/or the co-rotating interaction region of the solar wind (e.g. Burlaga, 1984 [ए13]).

\section{Conclusion}

We have analyzed the low-energy electrons measured by CALET from October 2015 to April 2019, and have succeeded to obtain the time profile of the normalized flux of the low-energy electrons with the energy from $1 \mathrm{GeV}$ to $10 \mathrm{GeV}$. We confirmed that the variation of the low-energy electrons measured by CALET is consistent with that expected from the recent solar cycle. Further investigation with continuous measurements of the low-energy electrons by CALET may provide a crucial key to the understanding of the details of the 27-day variation of the solar modulation (e.g. Kota and Jokipii, 2001 [ㅍ]]; Gil et al., 2005 [ए5]]). 


\section{References}

[1] O. Adriani et al. (CALET Collaboration), Phys. Rev. Lett., 119, 181101 (2017)

[2] O. Adriani et al. (CALET Collaboration), Phys. Rev. Lett., 120, 261102 (2018)

[3] O. Adriani et al. (CALET Collaboration), Phys. Rev. Lett. 122, 181102 (2019)

[4] Y. Asaoka, Y. Akaike, Y. Komiya, R. Miyata, S. Torii et al. (CALET Collaboration), Astropart. Phys. 91, 1 (2017)

[5] Y. Asaoka, Y. Ozawa, S. Torii et al. (CALET Collaboration), Astropart. Phys. 100, 29 (2018)

[6] K. Kasahara, in Proceeding of the 24th International Cosmic Ray Conference, Rome, Italy, 1, 399 (1995)

[7] D. J. Cooke, J. E. Humble, M. A. Shea et al., Nuovo Cimento C, 14 C, 3, 213 (1991)

[8] D. F. Smart, M. A. Shea, and E. O. Flúckiger, Space Sci. Rev., 93, 305-333 (2000)

[9] N. A. Tsyganenko, Planet. Space Sci., 37, 5-20 (1989)

[10] Y. Shikaze et al. (BESS Collaboration), Astropart. Phys., 28, 154 (2007)

[11] O. Adriani et al. (PAMELA Collaboration), Astrophys. J., 810, 142 (2015)

[12] M. Aguilar et al. (AMS-02 Collaboration), Phys. Rev. Lett., 121, 051102 (2018)

[13] L. F. Burlaga, Space Sci. Rev., 39, 255 (1984)

[14] J. Kota and J. R. Jokipii, Proceeding of the 27th International Cosmic Ray Conference, Hamburg, Germany, 9, 3577 (2001)

[15] A. Gil, K. Iskra, R. Modzelewska, and M. V. Alania, Adv. Space Rev., 35, 687 (2005) 